\title{
Optical elements for atoms: A beamsplitter and a mirror
}

\author{
M. Sigel, T. Pfau, C. S. Adams, C. Kurtsiefer, \\ W. Seifert, C. Heine, and J. Mlynek \\ Fakultăt für Physik, Universitāt Konstanz, D-78434 Konstanz, Deutschland \\ R. Kaiser and A. Aspect \\ Institut d' Optique, F-91403 Orsay, France
}

\section{Introduction}

Atom optics, in analogy to electron or neutron optics, is concerned with the manipulation of atomic matter waves [1-3]. Experiments on atomic beams bave been performed since the nineteen-twenties, but recently the improvement of tools, like free-standing microstructures and tunable laser light, has lead to a rapid development of the field. Beams of atoms bave been split coherently, focussed and reflected from mirrors. "Optical instruments" for atoms have a number of potential applications: Atom interferometers could be well suited as gravitational and inertial sensors and may allow precision measurements of atomic properties. A number of such measurements have already been carried out. An "atom-microscope" - investigating the interaction of atoms with surfaces with high spatial resolution - may provide an interesting new tool for surface physicists. "Atom lithography" may allow the deposition of atoms on surfaces with high precision. Cavities for atoms could be interesting to store cold atoms and for the study of quantum-statistical effects.

In the first part of this article we attempt to provide a very brief introduction to atom optics. In the second and third part we report on recent experiments in our group on two specific atom-optical elements that may be useful in atom interferometers and atom cavities: the demonstration of a new beam splitter based on the diffraction of atomic matter waves from a "magneto-optical grating" and the investigation of a mirror based on the reflection of atoms from an evanescent light field. 


\section{Atom optics}

Particle beams exhibit many phenomena well known from classical optics like diffraction and refraction. Particle optics based on electrons and neutrons [4] are well established fields. Experiments with these particles have played an important role in exploring the puzzling wave-particle duality. Both neutron and electron interferometers have been used in many beautiful experiments, which have improved our understanding of quantum mechanics. The interactions of neutron and electron beams with matter have become indispensable tools in the analysis of structures and surfaces, consider e.g. neutron scattering and the electron microscope.

As atoms are different from electrons and neutrons, particle optics with atoms promises a wealth of new effects and applications. Like neutrons, atoms are neutral and therefore cannot be manipulated as easily as electrons or ions using static electromagnetic fields. In return beams of atoms and neutrons are less susceptible to electromagnetic stray fields. Atoms are heavier, which makes them more suitable as gravitational and inertial sensors and means that very short de Broglie wavelengths can be obtained for lower particle energies. Atoms come in many species, which can be either bosons or fermions, and may have a total angular momentum or magnetic moment much larger than neutrons or electrons. Beams of atoms are easier and cheaper to produce than neutron beams as there is no need for a nuclear reactor. And maybe most importantly, atoms have a complex internal structure that can be probed and manipulated using resonant laser light or static electromagnetic fields.

As in classical optics, the building blocks of any experiment are optical elements e.g. beamsplitters, mirrors and lenses. Light can be manipulated by transmission through interfaces between materials with different refractive indices. Electrons, due to their charge, couple strongly to static electric or magnetic fields. Neutrons penetrate through solids and can be diffracted from crystal lattices. As atoms experience much smaller forces in static fields and do not penetrate through matter, none of these approaches are suitable for atom optics. 
So far optical elements for atoms have been demonstrated using diffraction, refraction or the recoil due to the stimulated emission or absorption of a single photon. "Diffractive optical elements" achieve the desired distortion of an incoming wave front by interaction with small structures, thus exploiting the wave nature of the atomic centre of mass motion. "Refractive optics" is based on introducing phase shifts by a spatial modulation of the index of refraction experienced by the atoms. In analogy to standard optics the index of refraction is defined as the ratio of a particle's group velocity and the vacuum group velocity. The index of refraction can be adjusted by shifting the potential energy of the atom. Intuitively this means: If the internal energy of an atom is e.g. decreased, energy conservation demands an acceleration of the centre of mass motion (i.e. an increase in the group velocity of the atomic matter wave). The phase velocity on the other hand is decreased in this case. The phase fronts are consequently retarded with respect to the unperturbed propagation. The principle of refractive optical elements is to choose the spatial dependence of these phase shifts to obtain the desired distortion of the incoming wave fronts. In static electric or magnetic fields the atomic internal energy can be changed by the Zeeman or Stark shift, in near-resonant light fields by the light shift.

The diffraction of atoms from the surface of single crystals was studied by Stern et al. as early as 1929 [5]. In 1969 Leavitt and Bills [6] observed a single-slit diffraction pattern using a thermal potassium beam. The progress in microfabrication technology now permits the production of structures sufficiently fine to diffract thermal atoms, whose de Broglie wavelengths are typically below $1 \dot{\mathrm{A}}$. The diffraction of thermal atoms from free-standing micro-fabricated gratings has been studied by several groups $[7,8]$. The first deflection of an atomic beam by the light pressure force was already demonstrated by Frisch in 1933 [9]. In recent years the availability of intense tunable laser radiation has again focussed interest on the mechanical effects of atom-ligbt interactions. The feasibility of such experiments has stimulated considerable theoretical interest. In addition to the conservative interaction using the dipole force, the spontaneous decay of excited electronic states can be utilized for the preparation of atomic beams by laser cooling techniques to obtain well-collimated, dense atomic beams with a well defined velocity. 
For small Rabi frequencies the levels are split by the Zeeman effect, for large Rabi frequencies by the light shift - for $\omega_{R}=2 \omega_{L}$ the Zeeman shift and the light shift at positions of circularly polarized light are equal and the eigenstate $|2\rangle$ displays the desired triangular modulation. For the V-system the ground state $|\mathrm{g}\rangle$ evolves adiabatically into this eigenstate $\mid 2$ ). If nonadiabatic processes and spontaneous emission do not populate the two other eigenstates the diffraction pattern obtained from this phase grating should display two distinct peaks.

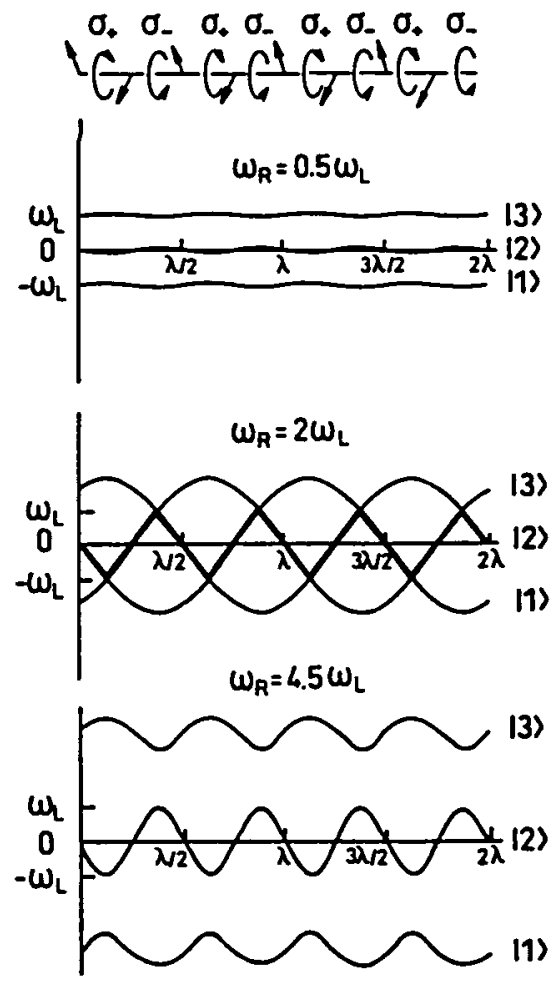

Figure 2: Plots of the spatial dependence of the eigenenergies of a three-level atom in a light field created by two counterpropagating orthogonally linear polarized light beams and a static magnetic field for three different ratios of Rabi frequency $\omega_{R}$ and Larmor frequency $\omega_{L}$. 


\subsection{Experiment}

We investigated the magneto-optical beamsplitter in an experiment using helium atoms in the triplet state. The light field was resonant with the transition at $1083 \mathrm{~nm}$ from the metastable $2^{3} \mathrm{~S}_{1}$ state to the $2^{3} \mathrm{P}_{1}$ state. If the incoming metastables are optically pumped into the $m_{\mathrm{J}}=0$ substate a $V$-type three-level system as depicted in figure $1 \mathrm{a}$ is obtained. A schematic of our experimental setup is displayed in figure 3 . The laser beam, which was derived from a Ti:sapphire ring laser, was focussed to a waist of $21 \mu \mathrm{m}$ in the beam direction. The polarization of the back-reflected beam was rotated by double pass through a N/4-plate. As a consequence of the large mean velocity in the supersonic beam of $1900 \mathrm{~m} / \mathrm{s}$ and the long lifetime of the excited state of $100 \mathrm{~ns}$ it was possible to largely avoid spontaneous emission. The superimposed magnetic field was produced by a pair of Helmhotz coils. The far-field diffraction pattern of the supersonic helium beam was observed with a scanning slit detector.

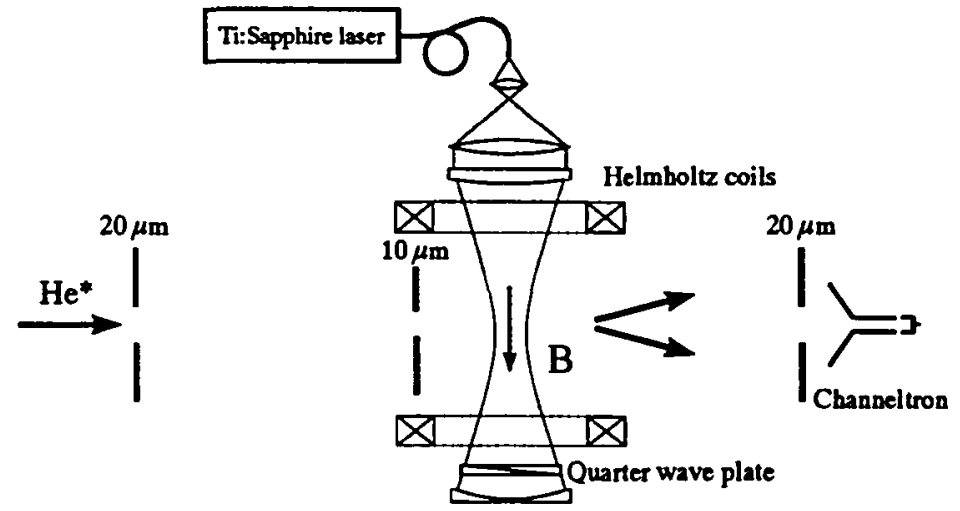

Figure 3: Experimental setup of the magneto-optical beamsplitter. A beam of triplet helium atoms interacted with two counterpropagating orthogonally linear polarized laser beams and a static magnetic field. The far-field diffraction pattern was detected with a scanning slit detector and a channeltron detector.

In Figure 4 the number of detected atoms is plotted as a function of transverse position in the detection plane $0.85 \mathrm{~m}$ downstream from the interaction zone. The choice of parameters was such that the Rabi frequency $\omega_{R}$ in the centre of the 
tends to decrease the coherence of the incoming beam due to the photon recoil as well as due to fluctuations in the dipole force. In order to minimize spontaneous emission by minimizing the interaction time, large gradients of the light intensity are desirable. Such large gradients can be obtained in evanescent light fields as suggested in 1982 by Cook and Hill [23]. Evanescent waves are created by total internal reflection of a light beam inside a dielectric medium; the electric field amplitude $E(z)$ in an evanescent wave produced by a plane wave of angular frequency $\omega$ for incidence angle $\varphi$ (measured against the surface plane) is given by

$$
\mathrm{E}(\mathrm{z})=\mathrm{E}(0) \cdot \mathrm{e}^{-\alpha \mathrm{z}}, \text { where } \alpha=\frac{\omega}{\mathrm{c}} \sqrt{\mathrm{n}^{2} \cos ^{2} \varphi-1} .
$$

If there is no spontaneous emission two-level atoms will be reflected in an evanescent light field of Rabi frequency $\omega_{R}$ and positive detuning $\Delta$ if the potential barrier $V(0)-V(\infty)\left(V_{ \pm}(z)= \pm \frac{\hbar}{2} \sqrt{\omega_{R}(z)^{2}+\Delta^{2}}\right)$ is larger than the transverse kinetic energy of the atoms $\frac{1}{2} m v \sin \Theta$. Spontaneous emission leads to statistical jumps between the two eigenstates of the atom and therefore to fluctuations of the force as well as a modified mean value (along with random photon recoil); in the steady state limit the mean force is given by the gradient of the eigenenergies weighted by the steady state population of the eigenstates [24].

In 1987 Balykin et al. reported such a reflection experiment using sodium atoms [25]. Kasevich et al. have demonstrated an "atomic trampoline" by bouncing slow sodium atoms off an evanescent wave under normal incidence [26]. Aminoff et al. recently have observed four bounces in this configuration using cesium atoms [27]. In our group we have investigated the reflection properties of an evanescent wave using metastable argon atoms.

\subsection{Reflection of metastable argon atoms from an evanescent wave}

In the first experiment we produced an evanescent wave on the surface of a glass prism in the setup shown in figure 6. The glass prism could be rotated to examine the reflection properties for various angles of incidence. A beam of metastable argon atoms in the metastable triplet state was directed on the glass prism such 


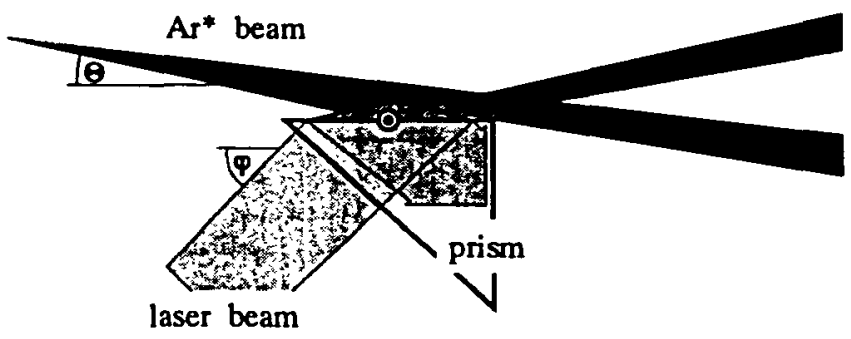

Figure 6: Schematic of the experimental setup for the reflection of atoms. The glass prism with the evanescent wave clipped the incoming atomic beam such that part of the beam passed the prism and part of the beam was reflected. The angle of incidence of the atomic beam $\theta$ could be adjusted by rotating the prism around the displayed axis.

that part of the beam passed the prism while part of the beam could interact with the evanescent wave. We used a channeltron detector, which is only sensitive to metastable states. The width of collimation slits and the width of the scanning slit were chosen such that the angular resolution was around $0.1 \mathrm{mrad}$. A Ti:sapphire ring laser was used to drive the two-level transition $1 \mathrm{~s}_{5}-2 \mathrm{p}_{9}$ (Paschen notation) at $812 \mathrm{~nm}$. The waists of the elliptical evanescent wave were $5.25 \mathrm{~mm}$ along the beam direction and $0.32 \mathrm{~mm}$ perpendicular to the beam direction. A set of experimental results for the atomic intensity as a function of the detector position for angles of incidence $\Theta$ between 0 and $3 \mathrm{mrad}$ are shown in figure 7. The peak at zero deflection angle is due to unreflected atoms which pass the prism. It can be seen that the reflection law is obeyed nicely and that there are no atoms scattered towards angles between the trespassing and the reflected beam. The decrease of the zero peak with increasing angle of incidence was caused by the increase of the "shadow" of the prism. The increasing area of the evanescent wave "seen" by the incoming beam led to the initial increase in the number of reflected atoms. For larger angles the increasing normal velocity led to a decrease in the area of the "Gaussian" evanescent wave where the intensity was large enough to deflect atoms. This effect caused the number of reflected atoms to ultimately drop to zero. 


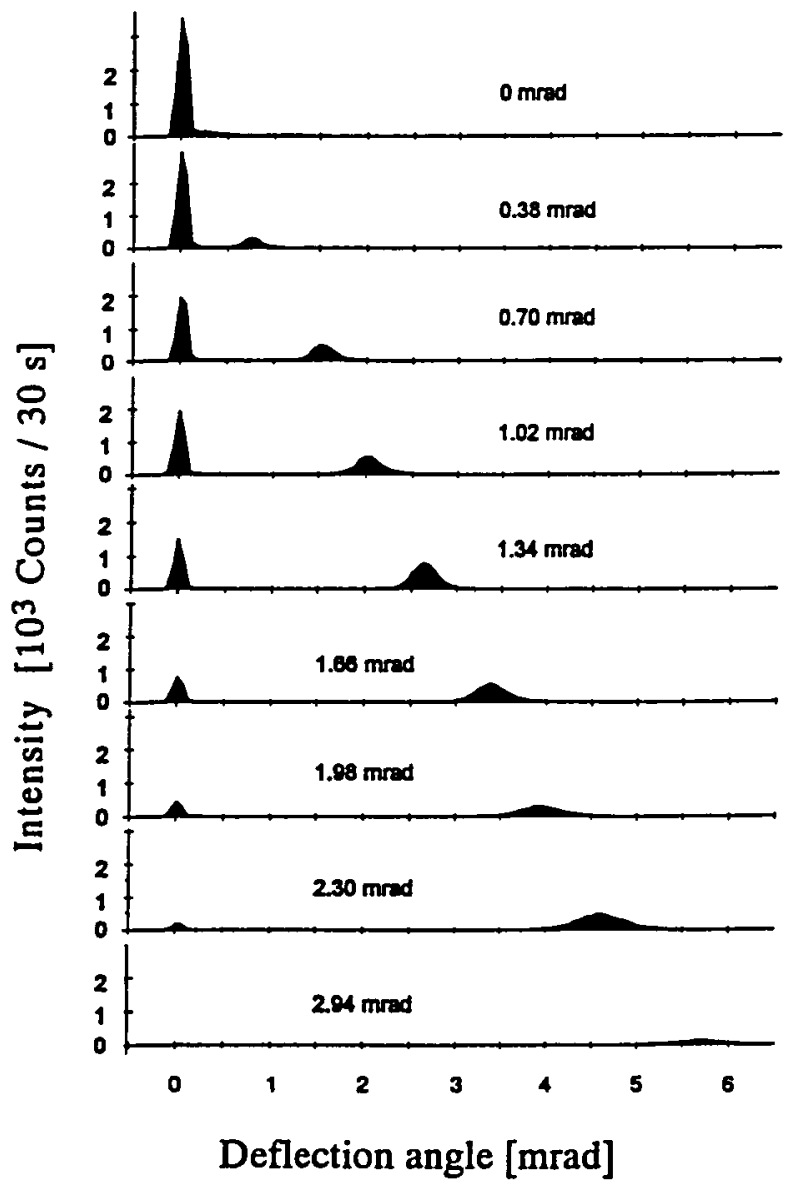

Figure 7: Experimental results for the number of detected metastable argon atoms over the detector position for various angles of incidence $\theta$ on the evanescent wave. The peaks at zero deflection angle are due to unreflected atoms which pass the glass prism while the right peaks are due to reflected atoms.

\subsection{Enhancements of evanescent waves using planar waveguides}

In order to reflect larger transverse velocities or in order to decrease the number of spontaneous emission by increasing the detuning very intense evanescent light waves are important. This is true despite the fact that the maximum transverse 


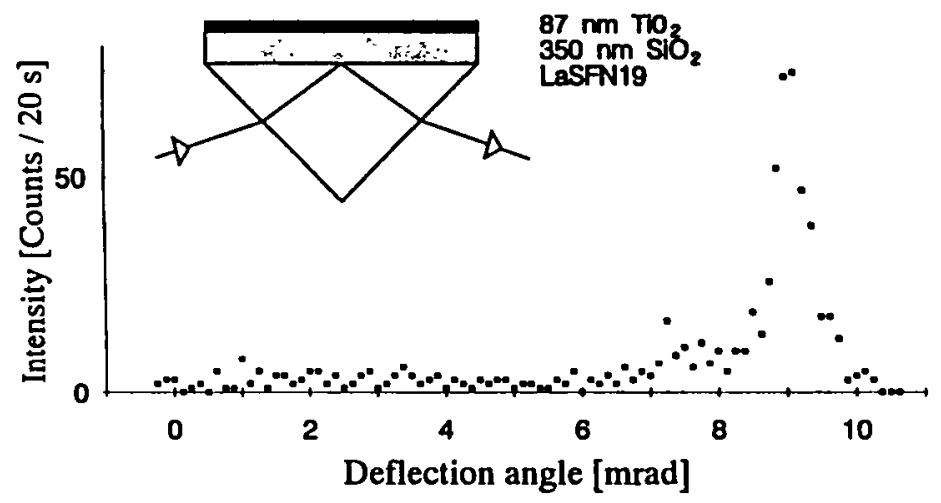

Figure 8: Experimental result for the reflection from an evanescent light field outside of a planar waveguide structure. The experimental configuration of the planar waveguide is displayed in the inset.

velocity and therefore the maximum angle of incidence scales only with the fourth root of the light intensity. The intensity of the evanescent field can be enhanced by resonantly increasing the light intensity at the surface. One possibility would be to use the surface as a mirror in a resonator. A specific type of such a resonator is a planar waveguide as shown in the inset of figure 8. In our experiment a $\mathrm{TiO}_{2}$ layer was used as the planar waveguide. The light was coupled in from a high index of refraction glass substrate $\left(\mathrm{LaSFN}_{18}\right)$ through a $\mathrm{SiO}_{2}$ spacer layer using frustrated total reflection. The evanescent field above the waveguide was enhanced by a factor of about 130 compared to the total internal reflection in the same prism for the same choice of polarization and angle of incidence (this is not the optimum choice of polarization for the reflection from a "bare" glass surface) [28].

We observed reflection of triplet argon atoms from this enhanced evanescent light field for angles of incidence $\Theta$ up to $6 \mathrm{mrad}$. This corresponds to transverse velocities of $3.4 \mathrm{~m} / \mathrm{s}$. An experimental result for the atomic intensity as a function of deflection angle is displayed in figure 8 . 


\subsection{Enhancements of evanescent waves using surface plasmons}

Another way to resonantly enhance the intensity of an evanescent wave is to excite surface plasmons in a metal film deposited on the surface of a glass substrate. Surface plasmons are surface waves of the electron gas in a metal that can be excited by reflecting light from a thin metal film. Reflection of atoms from plasmon enhanced evanescent waves very recently has been investigated by Feron et al. and Esslinger et al. [29,30]. In our experiment we used a $52 \mathrm{~nm}$ silver film on a glass substrate (Kretschmer configuration). For this configuration we again found reflection of atoms up to angles of incidence $\Theta$ of $6 \mathrm{mrad}$. An experimental result is displayed in figure 9.

We have performed numerical simulations on the reflection from the three types of evanescent waves. According to these simulations the transverse broadening of the reflected beams is largely due to fluctuations of the dipole force. The effect of the recoil in the spontaneous emission process is less important. The reflected beam is broader for the plasmon enhanced field than for the waveguide enhanced field because the "decay length" $\alpha$ of the evanescent wave was larger such that the interaction time was longer. In addition, variations in the intensity of the evanescent wave caused by variations in the thickness of the silver film may contribute to the broadening.

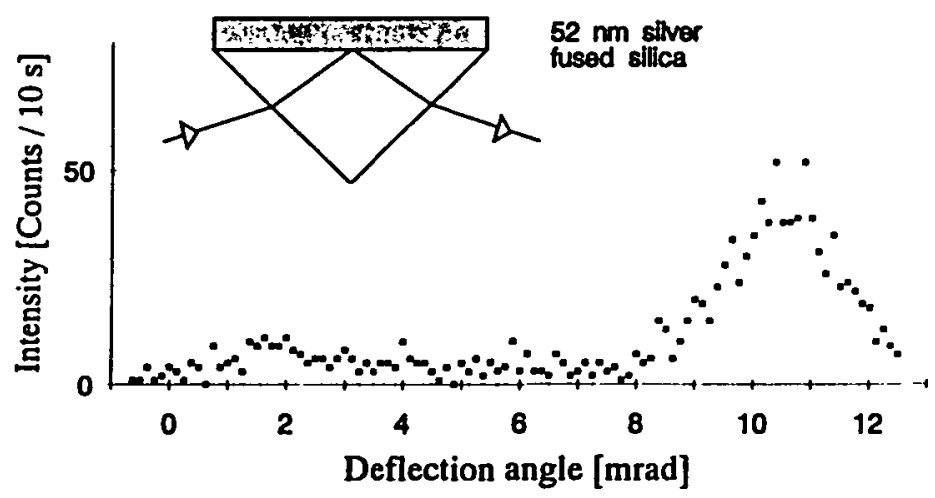

Figure 9: Experimental result for the reflection from an evanescent light field enhanced by surface plasmons on a silver film. A schematic of the glass prism with the silver film on top is displayed in the inset. 


\subsection{Estimate on the importance of spontaneous emission}

Spontaneous emission in the reflection process contributes to a decrease in the coherence of the incoming atomic matter wave. For applications like interferometers or cavities for atoms there must be no spontaneous emission. We investigated the importance of spontaneous emission by using the "open" transition $1 s_{5}-2 p_{8}$ at $802 \mathrm{~nm}$ in triplet argon. $71 \%$ of the atoms excited in the $2 \mathrm{p}_{8}$ state decay into the argon ground state, which is not detected by our channeltron detector. The number of detected reflected atoms compared to the result for a closed transition therefore contains information about the number of spontaneous emission processes. In figure 10 the maximum atomic intensity reflected from the plasmon enhanced evanescent wave is plotted as a function of the resonance detuning for both the "closed" and the "open" transition for the same angle of incidence of $1.1 \mathrm{mrad}$ and equal laser intensity. The detuning is given relative to the resonance frequency of the mean atonic velocity class. It can be seen that for the closed transition the reflected intensity rises from zero to a constant value for a positive detuning, the width of the rise being due to the finite velocity distribution in the atomic beam. For the open transition this rise is

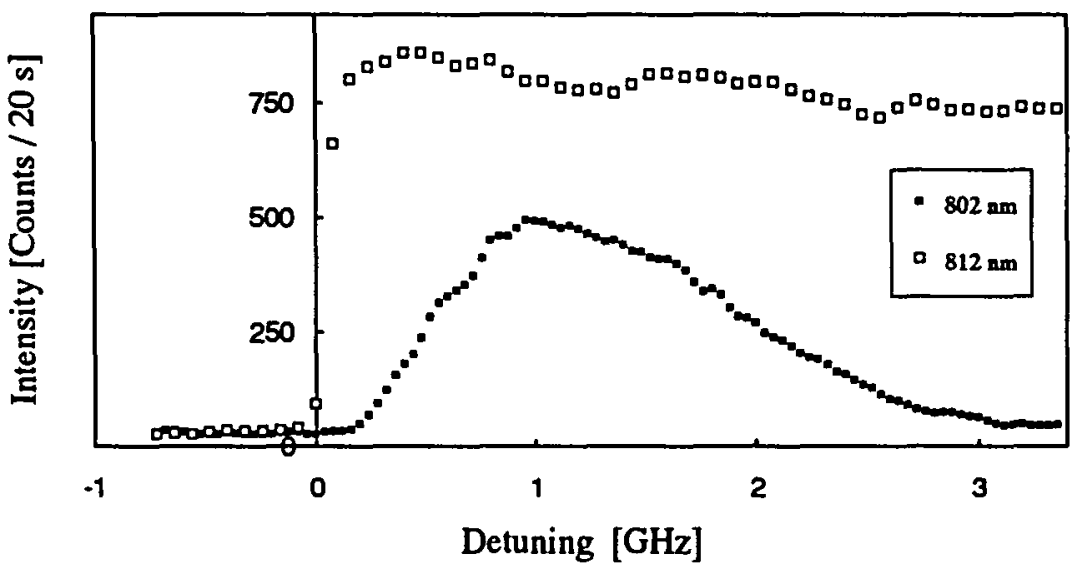

Figure 10: Plot of the reflected intensity as a function of the resonance detuning for the "closed" two level transition at $812 \mathrm{~nm}$ and the "open" transition at $802 \mathrm{~nm}$ in triplet argon. The "open" transtion allows part of the population of the excited state to decay to the argon ground state which is invisible to our detector. 
hampered by the decay into the undetected argon ground state such that the maximum is only reached for a detuning large enough to suppresses spontaneous emission. The decrease for large detunings sets in earlier than in case of the closed transition because the $812 \mathrm{~nm}$ transition is weaker. For the detuning at the maximum of this reflection curve the number of atoms reflected with no spontaneous emission can be estimated to larger than $90 \%$. This is a promising result in that it demonstrates that for a proper choice of experimental parameters the coherent reflection of atoms from an evanescent light field may be useful in atom interferometers and atom cavities.

\section{Conclusion}

In this contribution we presented experimental results for a new type of beamsplitter and an investigation of the reflecting properties of an evanescent light field. The beamsplitter relies on the diffraction of atomic matter waves from a phase grating with triangular phase modulation produced by a combination of a light field and a magnetic field. We observed a momentum splitting of $42 \hbar k$. In addition, we reported on the reflection of atoms from evanescent waves produced by total internal reflection of a laser beam. We demonstrated that the maximum reflection angle could be increased by enhancing the evanescent wave by a planar waveguide and surface plasmons. Furthermore, we examined the number of spontaneous emission processes in the reflection and concluded that for proper choice of parameters only few atoms will undergo spontaneous emission. A next step could be to investigate the coherence of these elements by combining them in a Mach-Zehnder type interferometer.

\section{Acknowledgement}

This work was supported by the Deutsche Forschungsgemeinschaft and the European Community through the SCIENCE programme under contract no. SC1-CT92-0778. 


\section{References}

[1] Special Issue of Appl. Phys. B (April 1992), Optics and Interferometry with Atoms, J. Mlynek, V. Balykin and P. Meystre (eds.).

[2] M. Sigel, C. S. Adams and J. Mlynek, to be published in Frontiers in LaserSpectroscopy, T. W. Hänsch, M. Inguscio (eds.), Proceedings of the International School of Physics "Enrico Fermi", Course CXX, Varenna, 1992.

[3] M. Sigel and J. Mlynek, Atom Optics, Physics World, February 1993.

[4] V. Sears, Neutron Optics (Oxford University Press, New York, Oxford, 1989).

[5] O. Stern, Naturwissensch. 17, 391 (1929).

[6] J. A. Leavitt and F. A. Bills, Am. J. Phys. 37, 905 (1969).

[7] D. W. Keith, M. L Schattenburg, H. I. Smith and D. E. Pritchard, Phys. Rev. Lett. 61, 1580 (1988).

[8] O. Carnal, A. Faulstich and J. Mlynek, Appl. Phys. B 53, 88 (1991).

[9] R. Frisch, Zeits. f. Phys. 86, 42 (1933).

[10] W. Gerlach und O. Stern, Zeits. f. Phys. 8, 110 (1922).

[11] T. Sleator, T. Pfau, V. Balykin, O. Carnal and J. Mlynek, Phys. Rev. Lett. 68, 1996 (1992).

[12] I. Estermann und O. Stern, Zeits. f. Phys. 61, 95 (1930).

[13] F. Riehle, Th. Kister, A. Witte, J. Helmcke and Ch. J. Bordé, Phys. Rev. Lett. 67, 177 (1991).

[14] M. Kasevich and S. Chu, Phys. Rev. Lett. 67, 181 (1991).

[15] P. E. Moskowitz, P. L Gould, S. R. Atlas and D. E. Pritchard, Phys. Rev. Lett. 51, 370 (1983).

[16] P. L Gould, G. A. Ruff and D. E. Pritchard, Phys. Rev. Lett. 56, 827 (1986).

[17] R. J. Cook and A. F. Bernhardt, Phys. Rev. A 18, 2533 (1978).

[18] T. Pfau, C. S. Adams and J. Mlynek, Europhys. Lett. 21, 439 (1993).

[19] C. S. Adams, T. Pfau, Ch. Kurtsiefer and J. Mlynek, "Interaction of atoms with a magneto-optical potential" to be published in Phys. Rev. A.

[20] F. Knauer und O. Stern, Zeits. f. Physik 53, 779 (1929).

[21] J. J. Berkhout, O. J. Luiten, I. D. Setija, T. W. Hijmans, T. Mizusaki and J. T. M. Walraven, Phys. Rev. Lett. 63, 1689 (1989).

[22] A. Anderson, S. Haroche, E. A. Hinds, W. Jhe, D. Meschede and L. Moi, Phys. Rev. A 34, 3513 (1986).

[23] R. J. Cook and R. K. Hill, Opt. Commun. 43, 258 (1982).

[24] J. Dalibard and C. Cohen-Tannoudji, J. Opt. Soc. Am. B 2, 1707 (1985).

[25] V. I. Balykin, V. S. Letokhov, Yu. B. Ovchinnikov and A. I. Sidorov, Phys. Rev. Lett. 60, 2137 (1988).

[26] M. A. Kasevich, D. S. Weiss and S. Chu, Opt. Lett. 15, 607 (1990).

[27] C. G. Aminoff, P. Bouyer and P. Desbiolles, C. R. Acad. Sc., Paris, 15 Mars 1993.

[28] R. Kaiser et al., Resonant enhancement of evanescent waves with a thin dielectric wave guide, submitted to Opt. Commun. 
[29] S. Feron et al., Reflection of metastable neon atoms by a surface plasmon wave, submitted to Europhys. Lett.

[30] T. Esslinger. M. Weidenmüller, A. Hemmerich and T. W. Hänsch, Opt. Lett. 18, 450 (1993). 